\title{
Recuperación de cartera bajo el enfoque de subconjuntos borrosos
}

\author{
Portfolio recovery under the blurred subset approach
}

Ximena Rocío González Astudillo. ${ }^{1}$, Kléber Antonio Luna Altamirano. ${ }^{2}$, Juan Carlos Erazo Álvarez. ${ }^{3}$, William Henry Sarmiento Espinoza. ${ }^{4}$

\begin{abstract}
DOI: https://doi.org/10.33262/cienciadigital.v3i2.3.595

Recovering a portfolio is one of the most difficult problems facing the Ecuadorian financial system, especially the Banco Diners Club in the city of Cuenca-Ecuador. The objective of this study is to provide this financial institution with advanced tools that offer fuzzy logic, with the purpose of trying to recover the portfolio caused by the use of their credit cards. Within the methodology, the technique of expertise is developed to feed a matrix of forgotten effects, which serves as a design means to structure a management strategy to face this problem and achieve a solution. It takes into consideration the actions stipulated by the financial institution at the time that there is a default of payments, the expertise is applied to then develop a convoluted matrix that can find the forgotten effects or omitted actions and must be considered by management in order to build the right path through a neural network and reach the recovery of portfolio, the hidden variable is home visit management, management may take into consideration this action and establish the necessary improvements in the collection management process.
\end{abstract}

Keywords: Forgotten effects, expertizaje, portfolio recovery, blurred subsets.

\section{Resumen}

Recuperar cartera es uno de los problemas más difíciles que enfrenta el sistema financiero ecuatoriano, muy en especial el Banco Diners Club de la ciudad de Cuenca-Ecuador. El objetivo de este estudio, es entregar a esta entidad financiera, herramientas de avanzada que ofrece la lógica difusa, con el propósito de tratar de recuperar la cartera ocasionada por el uso de sus tarjetas de crédito. Dentro de la metodología se desarrolla la técnica del expertizaje para alimentar una matriz de efectos olvidados, que sirve como medio de diseño para estructurar una estrategia de gestión para enfrentar este problema y lograr solucionar. Se toma en

\footnotetext{
${ }^{1}$ Universidad Católica de Cuenca, Maestrante en Administración de Empresas, Cuenca, Ecuador.xrgonzaleza536@psg.ucacue.edu.ec

${ }^{2}$ Universidad Católica de Cuenca, Unidad Académica de Administración, Cuenca, Ecuador.

klunaa@ucacue.edu.ec

${ }^{3}$ Universidad Católica de Cuenca, Posgrados, Cuenca, Ecuador. jcerazo@ucacue.edu.ec

${ }^{4}$ Universidad Católica de Cuenca, Unidad Académica de Administración, Cuenca, Ecuador. wsarmiento@ucacue.edu.ec
} 
consideración las acciones estipuladas por la institución financiera en el momento que se dé un incumplimiento de pagos, se aplica el expertizaje para luego elaborar una matriz convolucionada que permita encontrar los efectos olvidados o las acciones omitidas y que deben ser consideradas por la gerencia con la finalidad de construir el camino correcto mediante un grafo neuronal y poder llegar a la recuperación de cartera, la variable escondida resulta ser Gestión de visita domicilio, la gerencia podrá tomar en consideración esta acción y establecer la mejoras necesarias en el proceso de gestión de cobranzas.

Palabras clave: Efectos olvidados, expertizaje, recuperación de cartera, subconjuntos borrosos.

\section{Introducción}

La cartera es un problema para las instituciones financieras que operan en el mercado ofertando a los clientes créditos de diversas clases. En el caso del Banco Diners Club que ofrece tarjetas de crédito en la ciudad de Cuenca-Ecuador, el problema radica en la omisión de las obligaciones crediticias por parte de sus clientes, para lo cual se hace necesario generar una vía adecuada para el cumplimiento de este compromiso financiero, para ello esta institución financiera cuenta con acciones para recuperación de cartera, estipuladas dentro del Manual de Procedimientos de Gestión de Cobranzas canal Oficinas, no obstante a ello, se pretende generar una nueva estrategia de gestión con el propósito de afrontar este problema y tratar de resolverlo.

Este estudio presenta técnicas novedosas de la lógica difusa, como la teoría del expertizaje y efectos olvidados, desarrollados por Kaufmann y Gil Aluja (1989), con la finalidad de reducir la incertidumbre mediante la búsqueda de acciones omitidas por los expertos y que deben ser consideradas con la finalidad de tratar de recobrar estos impagos.

El objetivo de esta investigación es encontrar el camino propicio a través de un grafo neuronal, para poder llegar a recuperar esta cartera mediante el desarrollo de estas herramientas de avanzada que proporciona la lógica borrosa, con el propósito que la alta gerencia tome la correcta decisión en el momento adecuado.

\section{Estado del arte}

Las instituciones del sector financiero, atraviesan problemas de incumplimiento de pagos por créditos realizados, el riesgo de crédito está latente en el mercado financiero, por ello algunos autores dan a conocer sus investigaciones relacionados con este problema, entre ellos: López y Fuentes (2008) analizan la evolución, el cumplimiento y el índice de morosidad de la cartera de microcréditos del Sistema Bancario en Venezuela durante el periodo 2002-2005. Calderón y Castro (2013) explican las etapas planteadas en los lineamientos de la política bancaria referente al proceso de otorgamiento y recuperación del crédito, incorporando elementos que permitan mejorar el riesgo de recuperación del crédito en el sector agrícola. Chavarín (2015) explica que las restricciones de los créditos que mantiene la banca comercial mexicana es por el índice de morosidad de los prestatarios, el cual analiza mediante un modelo estático con estimadores HausmanTaylor y un modelo de panel dinámico con estimadores Arellano-Bover/Blundell-Bond. Támara-Ayús, Aristizábal y Velásquez (2012) realizan un análisis del riesgo crediticio y 
como a través del esquema de transición se puede calcular la probabilidad de incumplimiento de un deudor frente a un acreedor para una institución financiera de Colombia, efectúan una comparación del cálculo de la pérdida esperada entre el modelo empleado por la institución financiera, el modelo de referencia de calificación comercial planteado por la Superintendencia Financiera de Colombia y el modelo encontrado bajo el esquema de matrices de transición.

Bermúdez, Segura y Vercher (2007) presentan dos modelos borrosos de selección de carteras basados en el uso de intervalos de medias para el cálculo del rendimiento y del riesgo de la cartera, el primero aplican números borrosos de tipo LR con funciones de referencia de la misma forma analizando la relación entre dos definiciones diferentes de intervalos de medias; y el segundo emplean números borrosos trapezoidales efectuando la comparación de los modelos propuestos, que permitan seleccionar una cartera mediante la resolución de un problema de optimización lineal. Cardona (2006) demuestra a través de la teoría de portafolios, que en Colombia es posible utilizar el principio de la diversificación para reducir el riesgo en las carteras de acreditados, asignando los créditos de la banca empresarial, entre sectores con bajas correlaciones entre sí.

El profesor Lotfi Zadeh, considerado creador de la lógica difusa, en su obra "Fuzzy Sets" publicación que se lo hizo en 1975, dio a conocer los cimientos matemáticos anexados a la teoría de conjuntos difusos gracias a esta contribución se da inicio a la lógica difusa.

Ciertos autores han dado su contribución con respectos a la teoría de efectos olvidados, como herramienta de avanzada de la lógica borrosa, entre ellos: Salazar (2012) desarrolla un modelo no lineal para la predicción del comportamiento del tipo de cambio a futuro basado en la opinión de expertos, estas opiniones son tratadas mediante la teoría de efectos olvidados de la lógica borrosa. Gento, Lazzari y Machado (2001) dan a conocer sus experiencias en la aplicación de la metodología de recuperación de efectos olvidados en diferentes problemas de gestión, presentan algunas reflexiones sobre su utilización, sobre los efectos de orden mayor que dos, acerca de la incidencia del tiempo si se considera un proceso dinámico, a más de ello definen la estabilidad estricta y no estricta de una matriz de incidencia.

Rico y Tinto (2010) proponen la utilización de herramientas desarrolladas con base en la teoría de los subconjuntos borrosos, como el expertizaje-contraexpertizaje, y la teoría de los efectos olvidados en el tratamiento ex post de la información contable tradicional, con el fin de mejorar su capacidad para sustentar la toma de decisiones adecuadas a mediano y largo plazo. Tinto, Luna y Cisneros (2017) explican la teoría de efectos olvidados a través de variables escondidas que no son fáciles de detectar por el artesano y que deben tomarse en cuenta, ya que afectan la comercialización y permiten el rescate de esta actividad.

La lógica difusa, permite utilizar conceptos relativos de la realidad, definiendo grados variables de pertenencia y siguiendo patrones de razonamiento similares a los del pensamiento humano (Kosko, 1995). Otros autores, han dado un aporte a sus investigaciones a través de la lógica difusa. Kaufmann y Gil (1987) quienes aportaron con su valioso conocimiento de la lógica difusa a través de su obra Técnicas Operativas de Gestión para el Tratamiento de la Incertidumbre, definiendo a un número borroso como una secuencia finita o infinita de intervalos de confianza. Reig y González (2002) afirman: "la lógica borrosa se revela como un instrumento muy potente (...) al permitir, 
por un lado, recoger la incertidumbre generada por el entorno de la empresa, y por otro tratar la subjetividad que implica toda opinión de expertos" (p.436). Kaufmann y Gil Aluja, (1986) aseveran el uso de números borrosos triangulares en el tratamiento de la incertidumbre en la empresa es conocido desde los inicios de la incorporación de la lógica fuzzy en los problemas empresariales.

Casanovas y Fernández (2003) en su obra la gestión de la tesorería en la incertidumbre, desarrollan las herramientas de avanzada que brinda la lógica borrosa a modo de metodología, referente al recobro de impagos, problema que tienen las organizaciones con la finalidad de mejorar su gestión empresarial.

Los autores descritos, han fijado sus estudios en problemas de incumplimientos de pagos o morosidad por parte de clientes de distintas instituciones financieras, por ello la teoría de la lógica difusa ha sido ampliamente aplicado para tratar de solucionar problemas de gestión financiera, permitiendo demostrar elementos imprecisos e inexactos dentro de la toma de decisión gerencial.

\section{Metodología}

El desarrollo de la primera parte del estudio, es considerar las acciones (variables) para recuperar la cartera, mismas están establecidas dentro del Manual de Procedimientos de Gestión de Cobranzas canal Oficinas, estas se detallan a continuación:

Tabla 1 Acciones para la recuperación de cartera

\begin{tabular}{cl}
\hline SIMBOLOGIA & \multicolumn{1}{c}{$\begin{array}{c}\text { ACCIONES RECUPERACIÓN DE } \\
\text { CARTERA }\end{array}$} \\
\hline I & Pago en mora \\
II & Gestión telefónica SMS \\
III & Gestión telefónica primera llamada \\
IV & Gestión telefónica segunda llamada \\
V & Gestión telefónica tercera llamada \\
VI & Gestión visita a domicilio \\
VII & Gestión notificación carta refinanciación \\
VIII & Gestión notificación previa traslado judicial \\
& Gestión segunda notificación previa \\
IX & demanda \\
X & Traslado al área legal \\
\hline
\end{tabular}

Fuente: Elaboración propia

Entre las acciones determinadas en la tabla 1, se pretende encontrar las acciones o variables escondidas que permitan recuperar la cartera resultante de la utilización de la tarjeta de crédito, para ello se requiere la aplicación de herramientas de vanguardia como la teoría del expertizaje y de efectos olvidados, con el propósito de reducir la incertidumbre en la toma de decisiones a nivel gerencial. 


\section{Teoría del expertizaje}

A través de las herramientas de avanzada, se procura una mejor gestión empresarial, aplicando la teoría del expertizaje se trata de reducir la incertidumbre para una serie de datos o valores. Luna, Sarmiento y Cisneros (2017) aseveran: "Se entiende por expertizaje al proceso de consulta a un grupo determinado de expertos en relación con un tema definido, con el propósito de acotar la incertidumbre" (p.5). Kaufmann y Gil-Aluja (1989) afirman: "La introducción de una valuación matizada entre 0 y 1 permite hacer intervenir niveles de verdad en la noción de incidencia. (...) Valores de 0 a 1 (la llamada valuación endecadaria) (p. 26). La escala en mención se detalla en la tabla 2.

Tabla 2 Escala endecadaria

\begin{tabular}{cl}
$\begin{array}{c}\text { GRADO DE } \\
\text { PRESUNCIÓN }\end{array}$ & \multicolumn{1}{c}{ INCIDENCIA } \\
$\boldsymbol{\alpha}$ & \\
\hline 0 & Recuperación de cartera nulo \\
0,1 & Recuperación de cartera parcialmente nulo \\
0,2 & Recuperación de cartera muy cercano al nulo \\
0,3 & Recuperación de cartera mediamente cercano al nulo \\
0,4 & Recuperación de cartera cercano al nulo \\
0,5 & Recuperación de cartera ni total ni nulo \\
0,6 & Recuperación de cartera cercano al total \\
0,7 & Recuperación de cartera mediamente cercano al total \\
0,8 & Recuperación de cartera muy cercano al total \\
0,9 & Recuperación de cartera parcial total \\
1 & Recuperación de cartera total \\
\hline
\end{tabular}

Fuente: Elaboración propia

Se recurre a seis expertos en el área de crédito, quienes basándose en la escala endecadaria suministran información relacionada a la incidencia de las acciones entre ellas mismas, el resultado de incidencia de la acción "Pago en mora" sobre la "Gestión telefónica SMS", es la siguiente: Experto \# 1: 0,9; Experto \# 2: 0,5; Experto \# 3: 1; Experto \# 4: 0,9; Experto \# 5: 1; Experto \# 6: 1.

De las opiniones de los expertos, se observa que 0,9 se repite dos veces y 1,0 se repiten tres veces. Se registra el número de repeticiones con relación a la escala endecadaria sobre las opiniones de los seis expertos, con relación a la primera pregunta. El siguiente paso es normalizar la serie, esta consiste en dividir los valores de frecuencia obtenidos cada grado de presunción de la escala endecadaria entre el número de expertos (6), de la siguiente manera: $1 \div 6=0,17 ; 2 \div 6=0,33 ; y, 3 \div 6=0,50$, así sucesivamente. Se procede con la acumulación desde el valor final de la serie deteniéndose cuando se obtenga el valor de la unidad, de ahí en adelante todos los valores serán uno. El último paso es la sumatoria de la acumulación de frecuencias, no se considera el valor del grado de presunción $\alpha$ igual a cero. Lo explicado se detalla en la siguiente tabla: 
Tabla 3 Serie normalizada y acumulación de frecuencias

\begin{tabular}{|c|c|c|c|}
\hline $\begin{array}{c}\text { GRADO DE } \\
\text { PRESUNCIÓN } \\
\alpha\end{array}$ & FRECUENCIA & $\begin{array}{c}\text { NORMALIZACIÓN } \\
\text { DE LA } \\
\text { FRECUENCIA }\end{array}$ & $\begin{array}{c}\text { ACUMULACIÓN } \\
\text { DE } \\
\text { FRECUENCIAS }\end{array}$ \\
\hline 0 & 0 & 0 & 1 \\
\hline 0,1 & 0 & 0 & 1 \\
\hline 0,2 & 0 & 0 & 1 \\
\hline 0,3 & 0 & 0 & 1 \\
\hline 0,4 & 0 & 0 & 1 \\
\hline 0,5 & $1 / 6$ & 0,17 & 1 \\
\hline 0,6 & 0 & 0 & 0,83 \\
\hline 0,7 & 0 & 0 & 0,83 \\
\hline 0,8 & 0 & 0 & 0,83 \\
\hline 0,9 & $2 / 6$ & 0,33 & 0,83 \\
\hline 1 & $3 / 6$ & 0,50 & 0,50 \\
\hline TOTAL & 6 & 1,00 & 8,8 \\
\hline
\end{tabular}

Fuente: Elaboración propia

El resultado total obtenido de la sumatoria de la acumulación de frecuencias, se divide entre 10 correspondiente al número de factores que forman el grado de presunción $\alpha$ considerado desde 0,1 hasta 1 , es decir $8,8 \div 10=0,88$. Este mismo procedimiento se desarrolla para el resto de acciones, dando como resultado la matriz denominada como "A".

Tabla 4 Matriz de incidencia

\begin{tabular}{|c|c|c|c|c|c|c|c|c|c|c|c|}
\hline $\mathbf{A}$ & 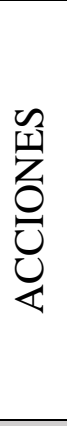 & 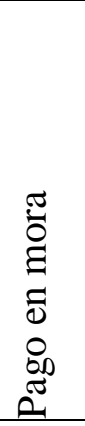 & 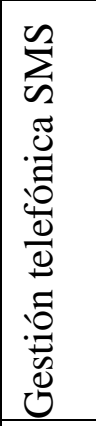 & 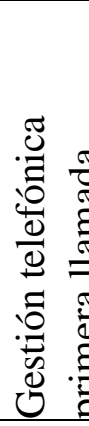 & 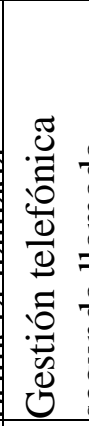 & 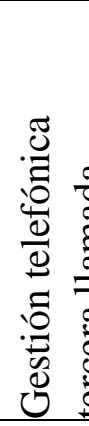 & 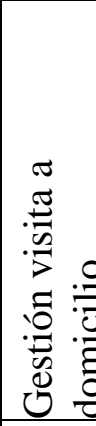 & 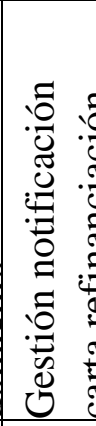 & 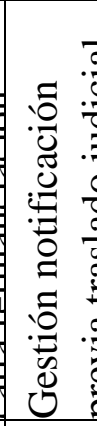 & 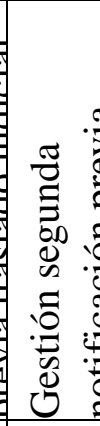 & 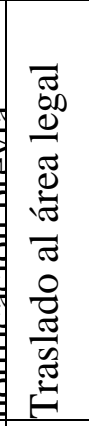 \\
\hline ACCIONES & & I & II & III & IV & $\mathrm{V}$ & VI & VII & VIII & IX & $X$ \\
\hline Pago en mora & I & 1 & $\mathbf{0 , 8 8}$ & 0,85 & 0,63 & 0,62 & 0,82 & 0,68 & 0,63 & 0,47 & 0,32 \\
\hline $\begin{array}{l}\text { Gestión telefónica } \\
\text { SMS }\end{array}$ & II & 0,73 & 1 & 0,75 & 0,63 & 0,57 & 0,62 & 0,52 & 0,47 & 0,42 & 0,27 \\
\hline $\begin{array}{l}\text { Gestión telefónica } \\
\text { primera llamada }\end{array}$ & III & 0,88 & 0,58 & 1 & 0,63 & 0,62 & 0,78 & 0,68 & 0,65 & 0,42 & 0,25 \\
\hline $\begin{array}{l}\text { Gestión telefónica } \\
\text { segunda llamada }\end{array}$ & IV & 0,70 & 0,53 & 0,63 & 1 & 0,62 & 0,62 & 0,57 & 0,52 & 0,50 & 0,38 \\
\hline
\end{tabular}




\begin{tabular}{|l|c|c|c|c|c|c|c|c|c|c|c|}
\hline $\begin{array}{l}\text { Gestión telefónica } \\
\text { tercera llamada }\end{array}$ & $\mathrm{V}$ & 0,63 & 0,47 & 0,53 & 0,53 & 1 & 0,63 & 0,60 & 0,60 & 0,52 & 0,43 \\
\hline $\begin{array}{l}\text { Gestión visita a } \\
\text { domicilio }\end{array}$ & $\mathrm{VI}$ & 0,60 & 0,42 & 0,62 & 0,62 & 0,62 & 1 & 0,72 & 0,68 & 0,73 & 0,80 \\
\hline $\begin{array}{l}\text { Gestión notificación } \\
\text { carta refinanciación }\end{array}$ & $\mathrm{VII}$ & 0,50 & 0,35 & 0,47 & 0,53 & 0,52 & 0,52 & 1 & 0,80 & 0,75 & 0,72 \\
\hline $\begin{array}{l}\text { Gestión notificación } \\
\text { previa traslado } \\
\text { judicial }\end{array}$ & $\mathrm{VIII}$ & 0,60 & 0,45 & 0,42 & 0,48 & 0,52 & 0,67 & 0,60 & 1 & 0,82 & 0,82 \\
\hline $\begin{array}{l}\text { Gestión segunda } \\
\text { notificación previa } \\
\text { demanda }\end{array}$ & $\mathrm{IX}$ & 0,72 & 0,45 & 0,43 & 0,37 & 0,38 & 0,63 & 0,63 & 0,65 & 1 & 0,83 \\
\hline Traslado al área legal & $\mathrm{X}$ & 0,72 & 0,55 & 0,43 & 0,43 & 0,47 & 0,62 & 0,67 & 0,72 & 0,90 & 1 \\
\hline
\end{tabular}

Fuente: Elaboración propia

\section{Teoría de efectos olvidados}

La teoría de efectos olvidados estudia aquellas variables no consideradas por los expertos durante el análisis de determinado tema referente a su relación causa-efecto. A través de la matriz de efectos olvidados se establece la incidencia o números borrosos con una valoración de $[0,1]$ determinada en una escala semántica o endecadaria, siendo a 1 la máxima importancia y 0 sin importancia (Kaufmann y Gil-Aluja, 1989), ésta escala endecadaria se obtuvo del proceso de expertizaje desarrollado anteriormente. Se demuestra la aplicación de la teoría de efectos olvidados en forma detallada:

El primer paso radica en realizar la convolución max-min que se representa con el signo $\mathrm{O}$, este proceso consiste en encontrar el máximo valor de una serie de valores mínimos resultantes de la comparación entre filas por columnas de una determinada matriz. El presente estudio considera para su cálculo con una matriz cuadrada con un número igual de filas y columnas, convolucionandose a sí misma, como resultado se obtiene la matriz "B“, a modo de ejemplo, se indica la convolución entre I-II, para el caso de la convolución entre la misma variable el valor será siempre1.

Para I-II:

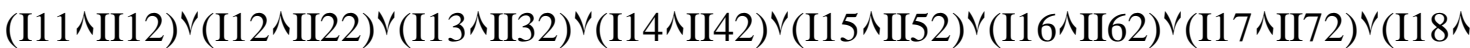
II82) $\vee($ I19^II92) $\vee($ I110^II102)

$(1,0 \wedge 0,88) \vee(0,88 \wedge 1,0) \vee(0,85 \wedge 0,58) \vee(0,63 \wedge 0,53) \vee(0,62 \wedge 0,47) \vee(0,82 \wedge 0,42)$ $\vee(0,68 \wedge 0,35) \vee(0,63 \wedge 0,45) \vee(0,47 \wedge 45) \vee(0,32 \wedge 0,55)$

Se elige el valor menor de cada par obtenido:

$0,88 \vee 0,88 \vee 0,58 \vee 0,53 \vee 0,47 \vee 0,42 \vee 0,35 \vee 0,45 \vee 0,45 \vee 0,32$ 
De los diez resultados obtenidos, se selecciona el mayor $(0,88)$ y se ubica en la matriz

"B" en la coordenada de I con II, como se indica en la tabla 5.

Tabla 5 Matriz convolucionada "B"

\begin{tabular}{|c|c|c|c|c|c|c|c|c|c|c|c|}
\hline B & 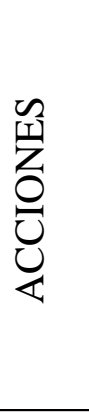 & 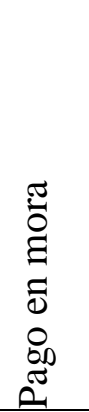 & 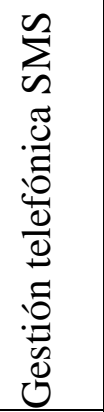 & 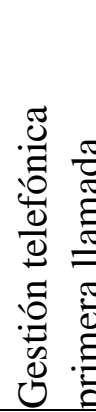 & 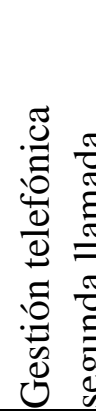 & 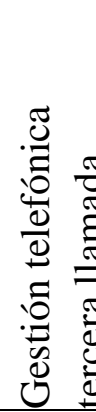 & 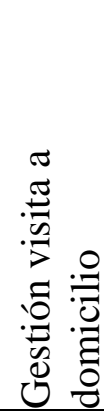 & 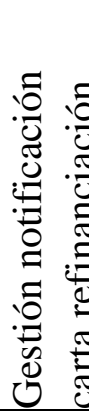 & 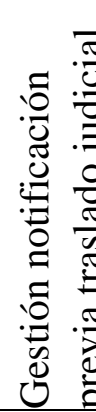 & 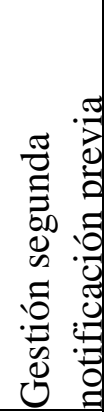 & 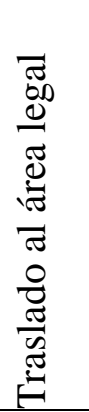 \\
\hline ACCIONES & & $\mathrm{I}$ & II & III & IV & V & VI & VII & VIII & IX & $X$ \\
\hline Pago en mora & I & 1 & 0,88 & 0,85 & 0,63 & 0,62 & 0,82 & 0,72 & 0,68 & 0,73 & 0,80 \\
\hline Gestión telefónica SMS & II & 0,75 & 1 & 0,75 & 0,63 & 0,62 & 0,75 & 0,68 & 0,65 & 0,62 & 0,62 \\
\hline $\begin{array}{l}\text { Gestión telefónica } \\
\text { primera llamada }\end{array}$ & III & 0,88 & 0,88 & 1 & 0,63 & 0,62 & 0,82 & 0,72 & 0,68 & 0,73 & 0,78 \\
\hline $\begin{array}{l}\text { Gestión telefónica } \\
\text { segunda llamada }\end{array}$ & IV & 0,70 & 0,70 & 0,70 & 1 & 0,62 & 0,70 & 0,68 & 0,63 & 0,62 & 0,62 \\
\hline $\begin{array}{l}\text { Gestión telefónica } \\
\text { tercera llamada }\end{array}$ & V & 0,63 & 0,63 & 0,63 & 0,63 & 1 & 0,63 & 0,63 & 0,63 & 0,63 & 0,63 \\
\hline $\begin{array}{l}\text { Gestión visita a } \\
\text { domicilio }\end{array}$ & VI & 0,72 & 0,60 & 0,62 & 0,62 & 0,62 & 1 & 0,72 & 0,72 & 0,80 & 0,80 \\
\hline $\begin{array}{l}\text { Gestión notificación } \\
\text { carta refinanciación }\end{array}$ & VII & 0,72 & 0,55 & 0,53 & 0,53 & 0,53 & 0,67 & 1 & 0,80 & 0,80 & 0,80 \\
\hline $\begin{array}{l}\text { Gestión notificación } \\
\text { previa traslado judicial }\end{array}$ & VIII & 0,72 & 0,60 & 0,62 & 0,62 & 0,62 & 0,67 & 0,67 & 1 & 0,82 & 0,82 \\
\hline $\begin{array}{l}\text { Gestión segunda } \\
\text { notificación previa } \\
\text { demanda }\end{array}$ & IX & 0,72 & 0,72 & 0,72 & 0,63 & 0,62 & 0,72 & 0,68 & 0,72 & 1 & 0,83 \\
\hline Traslado al área legal & $X$ &, 72 & 0,72 & 0,72 & 0,63 & 0,62 & 0,72 & 0,68 & 0,72 & 0,90 & 1 \\
\hline
\end{tabular}

Fuente: Elaboración propia

La matriz "B" es considerada como aquella que contiene los efectos olvidados de primera generación, esta se coteja con la matriz original " $\mathrm{A}$ " como se observa en la tabla 6; se determina los " $\alpha$ " datos que indiquen las mayores diferencias al restar los cuadrantes coincidentes de B-A así: B (I,I)-A(I,I), B(I,II)-A(I,II), B(I,II)-A(I,II), y así sucesivamente. 
Tabla 6 Comparación de matrices para la obtención de efectos olvidados

\begin{tabular}{|c|c|c|c|c|c|c|c|c|c|c|c|}
\hline B & 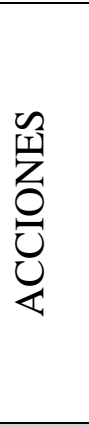 & 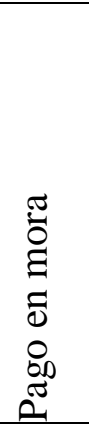 & 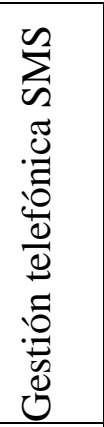 & 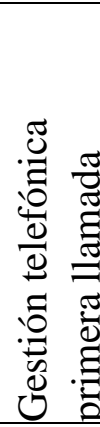 & 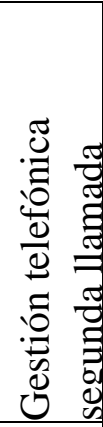 & 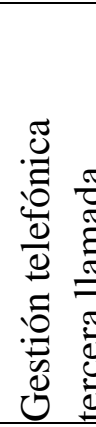 & 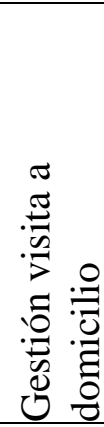 & 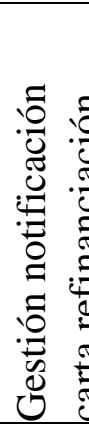 & 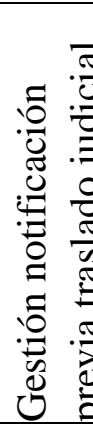 & 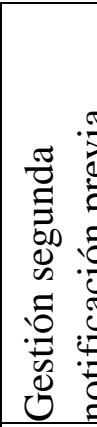 & 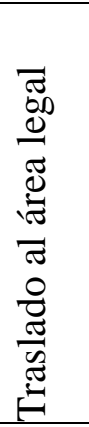 \\
\hline ACCIONES & & I & II & III & IV & $\mathrm{V}$ & VI & VII & VIII & IX & $\mathrm{X}$ \\
\hline Pago en mora & I & 1 & 0,88 & 0,85 & 0,63 & 0,62 & 0,82 & 0,72 & 0,68 & 0,73 & 0,80 \\
\hline Gestión telefónica SMS & II & 0,75 & 1 & 0,75 & 0,63 & 0,62 & 0,75 & 0,68 & 0,65 & 0,62 & 0,62 \\
\hline $\begin{array}{l}\text { Gestión telefónica } \\
\text { primera llamada }\end{array}$ & III & 0,88 & 0,88 & 1 & 0,63 & 0,62 & 0,82 & 0,72 & 0,68 & 0,73 & 0,78 \\
\hline $\begin{array}{l}\text { Gestión telefónica } \\
\text { segunda llamada }\end{array}$ & IV & 0,70 & 0,70 & 0,70 & 1 & 0,62 & 0,70 & 0,68 & 0,63 & 0,62 & 0,62 \\
\hline $\begin{array}{l}\text { Gestión telefónica } \\
\text { tercera llamada }\end{array}$ & V & 0,63 & 0,63 & 0,63 & 0,63 & 1 & 0,63 & 0,63 & 0,63 & 0,63 & 0,63 \\
\hline $\begin{array}{l}\text { Gestión visita a } \\
\text { domicilio }\end{array}$ & VI & 0,72 & 0,60 & 0,62 & 0,62 & 0,62 & 1 & 0,72 & 0,72 & 0,80 & 0,80 \\
\hline $\begin{array}{l}\text { Gestión notificación } \\
\text { carta refinanciación }\end{array}$ & VII & 0,72 & 0,55 & 0,53 & 0,53 & 0,53 & 0,67 & 1 & 0,80 & 0,80 & 0,80 \\
\hline $\begin{array}{l}\text { Gestión notificación } \\
\text { previa traslado judicial }\end{array}$ & VIII & 0,72 & 0,60 & 0,62 & 0,62 & 0,62 & 0,67 & 0,67 & 1 & 0,82 & 0,82 \\
\hline $\begin{array}{l}\text { Gestión segunda } \\
\text { notificación previa } \\
\text { demanda }\end{array}$ & IX & 0,72 & 0,72 & 0,72 & 0,63 & 0,62 & 0,72 & 0,68 & 0,72 & 1 & 0,83 \\
\hline Traslado al área legal & $\mathrm{X}$ & 0,72 & 0,72 & 0,72 & 0,63 & 0,62 & 0,72 & 0,68 & 0,72 & 0,90 & 1 \\
\hline
\end{tabular}

\begin{tabular}{|c|c|c|c|c|c|c|c|c|c|c|c|}
\hline A & 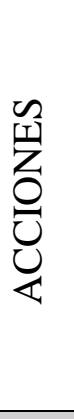 & 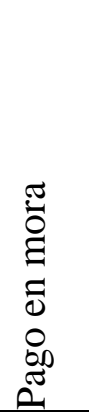 & 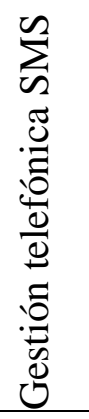 & 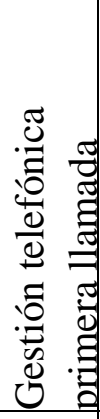 & 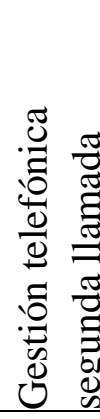 & 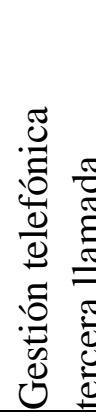 & 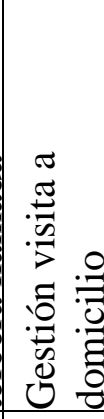 & 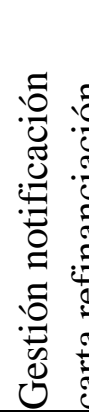 & 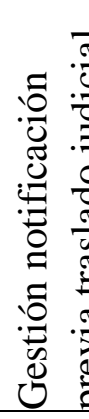 & 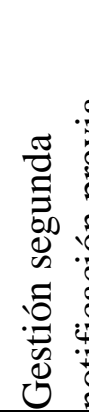 & 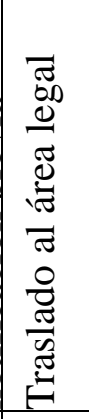 \\
\hline ACCIONES & & I & II & III & IV & V & VI & VII & VIII & IX & $X$ \\
\hline Pago en mora & I & 1 & $\mathbf{0 , 8 8}$ & 0,85 & 0,63 & 0,62 & 0,82 & 0,68 & 0,63 & 0,47 & 0,32 \\
\hline Gestión telefónica SMS & II & 0,73 & 1 & 0,75 & 0,63 & 0,57 & 0,62 & 0,52 & 0,47 & 0,42 & 0,27 \\
\hline
\end{tabular}


ISSN: 2602-8085

www.cienciadigital.org

Vol. 3, N².3., p. 156-171, abril - junio, 2019

\begin{tabular}{|l|c|c|c|c|c|c|c|c|c|c|c|}
\hline $\begin{array}{l}\text { Gestión telefónica } \\
\text { primera llamada }\end{array}$ & III & 0,88 & 0,58 & 1 & 0,63 & 0,62 & 0,78 & 0,68 & 0,65 & 0,42 & 0,25 \\
\hline $\begin{array}{l}\text { Gestión telefónica } \\
\text { segunda llamada }\end{array}$ & IV & 0,70 & 0,53 & 0,63 & 1 & 0,62 & 0,62 & 0,57 & 0,52 & 0,50 & 0,38 \\
\hline $\begin{array}{l}\text { Gestión telefónica } \\
\text { tercera llamada }\end{array}$ & V & 0,63 & 0,47 & 0,53 & 0,53 & 1 & 0,63 & 0,60 & 0,60 & 0,52 & 0,43 \\
\hline $\begin{array}{l}\text { Gestión visita a } \\
\text { domicilio }\end{array}$ & VI & 0,60 & 0,42 & 0,62 & 0,62 & 0,62 & 1 & 0,72 & 0,68 & 0,73 & 0,80 \\
\hline $\begin{array}{l}\text { Gestión notificación } \\
\text { carta refinanciación }\end{array}$ & VII & 0,50 & 0,35 & 0,47 & 0,53 & 0,52 & 0,52 & 1 & 0,80 & 0,75 & 0,72 \\
\hline $\begin{array}{l}\text { Gestión notificación } \\
\text { previa traslado judicial }\end{array}$ & VIII & 0,60 & 0,45 & 0,42 & 0,48 & 0,52 & 0,67 & 0,60 & 1 & 0,82 & 0,82 \\
\hline $\begin{array}{l}\text { Gestión segunda } \\
\text { notificación previa } \\
\text { demanda }\end{array}$ & IX & 0,72 & 0,45 & 0,43 & 0,37 & 0,38 & 0,63 & 0,63 & 0,65 & 1 & 0,83 \\
\hline Traslado al área legal & $\mathrm{X}$ & 0,72 & 0,55 & 0,43 & 0,43 & 0,47 & 0,62 & 0,67 & 0,72 & 0,90 & 1 \\
\hline
\end{tabular}

Fuente: Elaboración propia

Tabla 7 Matriz de resultados B - A

\begin{tabular}{|c|c|c|c|c|c|c|c|c|c|c|c|}
\hline $\mathbf{B}-\mathbf{A}$ & 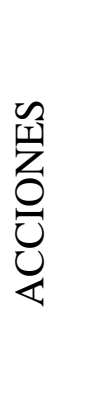 & 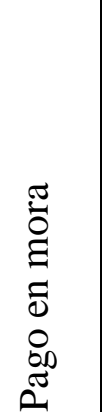 & 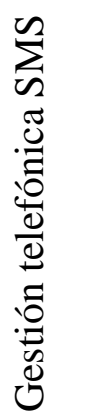 & 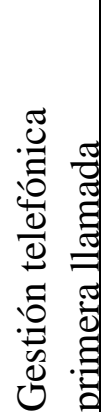 & 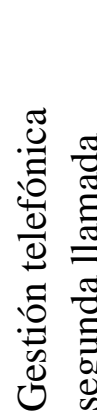 & 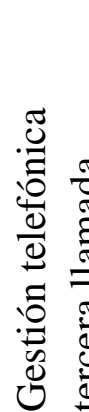 & 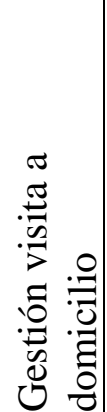 & 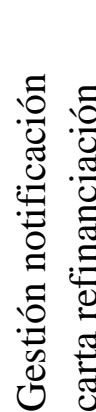 & 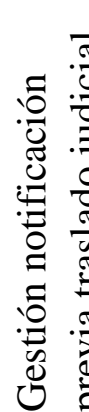 & 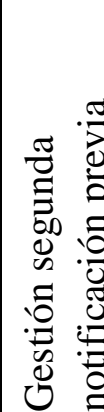 & 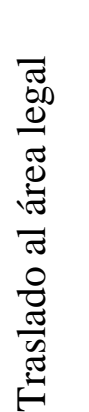 \\
\hline ACCIONES & & $\mathrm{I}$ & II & III & IV & $\mathrm{V}$ & VI & VII & VIII & IX & $\mathrm{X}$ \\
\hline Pago en mora & I & 0.00 & 0.00 & 0.00 & 0.00 & 0.00 & 0.00 & 0.03 & 0.05 & 0.27 & 0.48 \\
\hline Gestión telefónica SMS & II & 0.02 & 0.00 & 0.00 & 0.00 & 0.05 & 0.13 & 0.17 & 0.18 & 0.20 & 0.35 \\
\hline $\begin{array}{l}\text { Gestión telefónica } \\
\text { primera llamada }\end{array}$ & III & 0.00 & 0.30 & 0.00 & 0.00 & 0.00 & 0.03 & 0.03 & 0.03 & 0.32 & $\underline{0.53}$ \\
\hline $\begin{array}{l}\text { Gestión telefónica } \\
\text { segunda llamada }\end{array}$ & IV & 0.00 & 0.17 & 0.07 & 0.00 & 0.00 & 0.08 & 0.12 & 0.12 & 0.12 & 0.23 \\
\hline $\begin{array}{l}\text { Gestión telefónica } \\
\text { tercera llamada }\end{array}$ & V & 0.00 & 0.17 & 0.10 & 0.10 & 0.00 & 0.00 & 0.03 & 0.03 & 0.12 & 0.20 \\
\hline $\begin{array}{l}\text { Gestión visita a } \\
\text { domicilio }\end{array}$ & VI & 0.12 & 0.18 & 0.00 & 0.00 & 0.00 & 0.00 & 0.00 & 0.03 & 0.07 & 0.00 \\
\hline $\begin{array}{l}\text { Gestión notificación } \\
\text { carta refinanciación }\end{array}$ & VII & 0.22 & 0.20 & 0.07 & 0.00 & 0.02 & 0.15 & 0.00 & 0.00 & 0.05 & 0.08 \\
\hline
\end{tabular}




\begin{tabular}{|l|c|c|c|c|c|c|c|c|c|c|c|}
\hline $\begin{array}{l}\text { Gestión notificación } \\
\text { previa traslado judicial }\end{array}$ & VIII & 0.12 & 0.15 & 0.20 & 0.13 & 0.10 & 0.00 & 0.07 & 0.00 & 0.00 & 0.00 \\
\hline $\begin{array}{l}\text { Gestión segunda } \\
\text { notificación previa } \\
\text { demanda }\end{array}$ & IX & 0.00 & 0.27 & 0.28 & 0.27 & 0.23 & 0.08 & 0.05 & 0.07 & 0.00 & 0.00 \\
\hline Traslado al área legal & $\mathrm{X}$ & 0.00 & 0.17 & 0.28 & 0.20 & 0.15 & 0.10 & 0.02 & 0.00 & 0.00 & 0.00 \\
\hline
\end{tabular}

Fuente: Elaboración propia

Se restan los valores de los cuadrantes respetando las coordenadas de cada matriz, los datos resultantes se consideran en valor absoluto y se anotan en la matriz "B - A", por ejemplo la resta de B(I,I) - A(I,IA) (1 - 1) se obtiene 0,0, dato que se debe anotar en la matriz "B - A" en la intersección de I con I. De este resultado, se analiza los valores más cercanos a la unidad, estos representan mayor significado en términos de efectos olvidados. Para el presente estudio, se considera el valor " $\alpha$ " 0,53 , situado en la coordenada III-X, este es el que más se aproxima a la unidad, esto significa que se busca las acciones que se olvidaron u omitieron los expertos en la incidencia de la Gestión telefónica primera llamada sobre el Traslado al área legal.

Como siguiente paso de la teoría de efectos olvidados, se analiza cómo una variable influye sobre la otra, encontrando la acción que tiene incidencia de causalidad entre las dos acciones que se observan de primera mano.

En la misma posición que se encuentra el valor de " $\alpha$ " igual a 0,53 , en la coordenada (III, $\mathrm{X}$ ) de la matriz "B - A", se traslada a la matriz inicial "A". El procedimiento de la convolución max-min, se realiza nuevamente comparando los valores que denoten incidencia entre las acciones "III, X" de la tabla 4 (matriz A), que forman la fila - columna en esa intersección, de la siguiente manera.

Para III, X:

$(\text { III } 31 \wedge X 110)^{\vee}(\text { III } 32 \wedge X 210)^{\vee}(\text { III } 33 \wedge X 310)^{\vee}(\text { III } 34 \wedge X 410)^{\vee}($ III $35 \wedge X 510) \vee(I I I 36 \wedge X 610)$ $\vee($ III $37 \wedge X 710) \vee(I I I 38 \wedge X 810) \vee(I I I 39 \wedge X 910) \vee(I I I 310 \wedge X 910)$

$(0,88 \wedge 0,32(\vee(0,58 \wedge 0,27(\vee) 1,0 \wedge 0,25(\vee(0,63 \wedge 0,38(\vee(0,62 \wedge 0,43(\vee(0,78 \wedge$ $0,80) \vee(0,68 \wedge 0,72) \vee(0,65 \wedge 0,82) \vee(0,42 \wedge 0,83) \vee(0,25 \wedge 1,0)$

Se elige el valor menor de cada par obtenido:

$0,32 \vee 0,27 \vee 0,25 \vee 0,38 \vee 0,43 \vee 0,78 \vee 0,68 \vee 0,65 \vee 0,42 \vee 0,25$

Se opta por el mayor valor, este es 0,78 representa la máxima incidencia que tienen entre las acciones "III, X" sobre la acción "VI". La explicación completa se indica en el siguiente gráfico: 
Figura 1 Incidencia de la causalidad

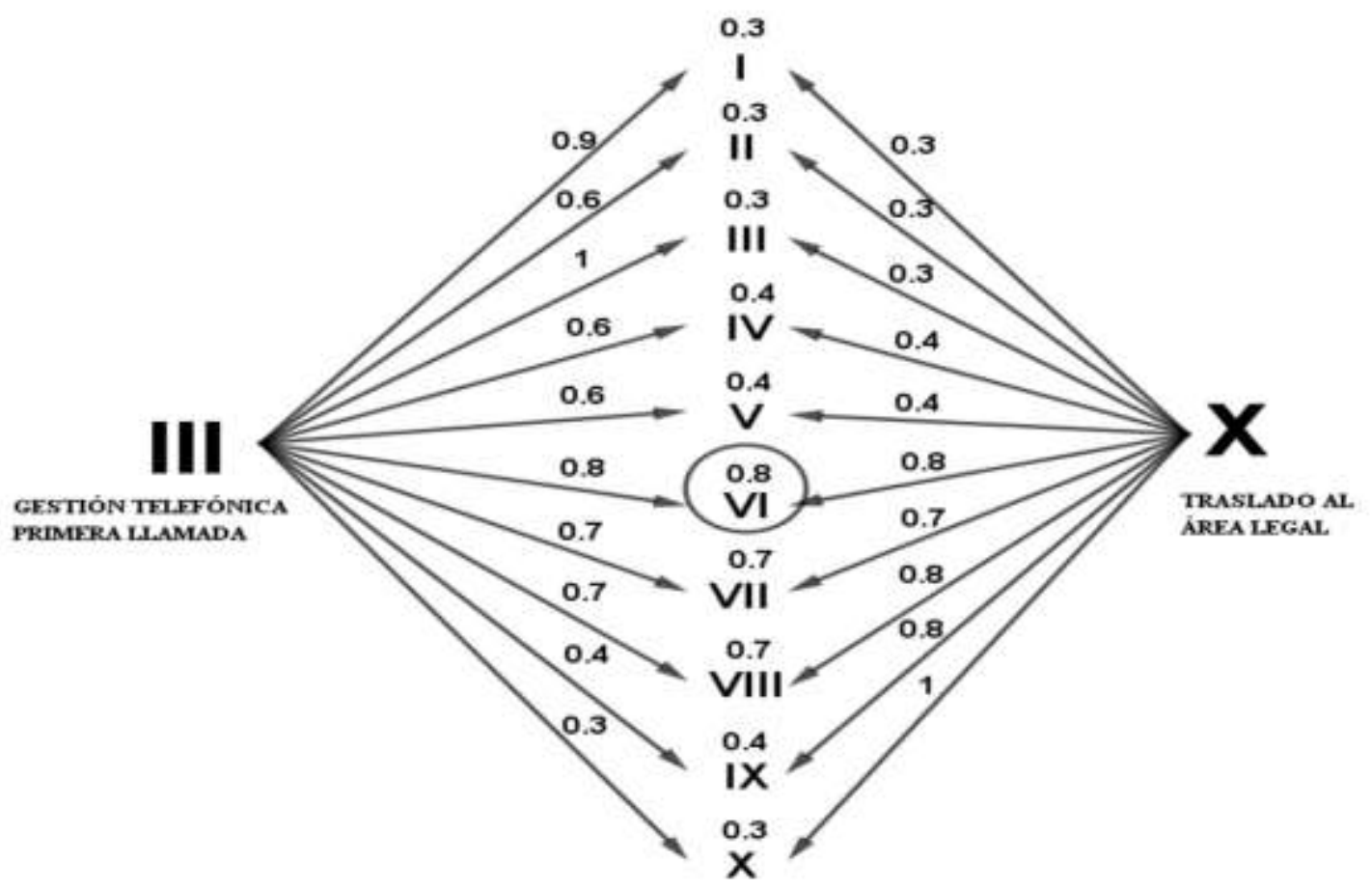

Fuente: Elaboración propia

El resultado, se lo interpreta de la siguiente manera: La Gestión telefónica primera llamada, incide sobre el Traslado al área legal a través de la Gestión visita a domicilio. Con la acción encontrada, se traza el camino correcto utilizando un grafo mediante una red neuronal, ésta constituye una herramienta propicia para la mejora en la gestión empresarial. Casanovas y Fernández (2003) manifiestan: "la teoría de grafos nos proporciona una amplia panoplia de herramientas para la gestión empresarial, especialmente cuando se trata de llevar a cabo un conjunto de acciones secuenciales para la resolución de un problema o consecución de un objetivo" (p.55). Las redes neuronales proporcionan la combinación de parámetros que se acoplan de mejor manera a la solución de problemas, estructurando capas o niveles compuestas por vértices de forma tal que al desplazarse de una a otra será imposible regresar a la anterior, se establecen asociaciones de vértices sistemáticos de manera lógica siguiendo un horizonte correcto. A partir de los resultados se diseña la vía exacta determinando los niveles o capas representativas, que conduzcan a la institución financiera a tratar de recuperar la cartera, considerando de manera prioritaria la acción olvidada, este recorrido da inicio con la acción Gestión telefónica primera llamada, cuya incidencia recae sobre el Traslado al área legal, a través de la Gestión visita a domicilio. El siguiente grafico demuestra lo enunciado. 
Figura 2 Grafo neuronal

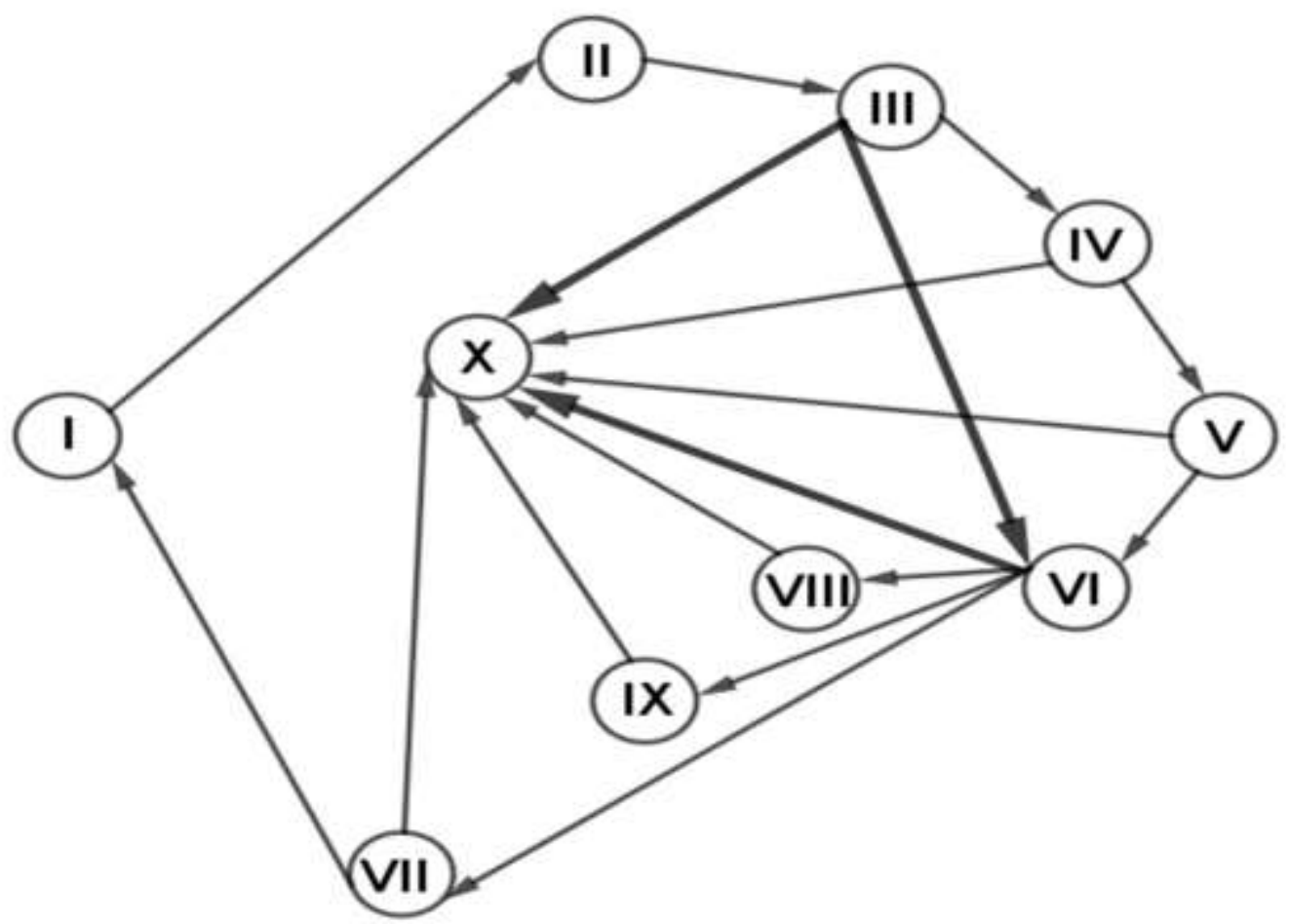

Fuente: Elaboración propia

\section{Resultados}

Una vez aplicada la teoría del expertizaje y efectos olvidados, el resultado determina que la Gestión telefónica primera llamada, incide sobre el Traslado al área legal a través de la variable escondida Gestión visita a domicilio. La gerencia deberá tomar en consideración esta acción olvidada, con el propósito de definir el proceso a seguir para lograr la recuperación de la cartera.

\section{Conclusiones}

La utilización de las técnicas de avanzada que ofrece la lógica difusa, demuestra su efectividad para la gestión de cobranzas en instituciones bancarias, permitiendo a los responsables seguir la ruta correcta mediante un grafo neuronal estableciendo las estrategias para una óptima recuperación de los pagos en mora, esta vía está compuesta por vértices que conforman una red neuronal, en donde la institución financiera encuentra el camino correcto para lograr dar solución a este problema, con ello se pretende general una política de cobro a través de variables escondidas u omitidas las cuales deben ser consideradas por la gerencia para enfrentar el riesgo financiero, tratando de reducir el problema de financiación a corto plazo, permitiendo definir modelos efectivos y eficientes en la toma de decisión gerencial. Con la gestión de visita a domicilio se pretende interactuar con el deudor y mantener la relación comercial a través de la recuperación de la cartera o generación de acuerdos de pago, caso contrario el Banco 
Diners Club puede trasladar el caso al área legal. De esta manera se entrega a la alta gerencia de la institución financiera, esta metodología como un aporte para una correcta toma de decisión en el proceso de gestión de cobranzas, con ello queda abierto la posibilidad para otras investigaciones desarrollando nuevas herramientas de la lógica borrosa en beneficio de este sector.

\section{Referencias bibliográficas}

Bermúdez, J., Segura, J., y Vercher, E. (2007). Modelos borrosos de optimización para la selección de carteras basados en intervalos de medias. Cuadernos del CIMBAGE, (9), 27-36. Recuperado de http://www.redalyc.org/articulo.oa?id=46200902

Calderón, M., y Castro, A. (2013). Alternativa metodológica para el otorgamiento y recuperación del crédito bancario en el BANDEC. Ciencias Holguín, XIX (4), 110. Recuperado de http://www.redalyc.org/articulo.oa?id=181529929005

Cardona, Z. (2006). La diversificación del riesgo en la cartera de créditos del sector financiero con base en la teoría de portafolios. AD-minister, (9), 113-136. Recuperado de http://www.redalyc.org/articulo.oa?id=322327239005

Chavarín, R. (2015). Morosidad en el pago de créditos y rentabilidad de la banca comercial en México. Revista Mexicana de Economía y Finanzas. Nueva Época / Mexican Journal of Economics and Finance, 10(1), 71-83. Recuperado de: http://www.redalyc.org/articulo.oa?id=423739513004

Gento, A., Lazzari, L., y Machado, E. (2001). Reflexiones acerca de las matrices de incidencia y la recuperación de efectos olvidados. Cuadernos del CIMBAGE, 4. 11-27. Recuperado de http://redalyc.org/articulo.oa?id=46200402

Kaufmann, A., y Gil-Aluja J. (1987). Técnicas operativas de gestión para el tratamiento de la incertidumbre. Barcelona-España: Hispano Europea.

Kaufmann, A., y Gil-Aluja, J. (1986). Introducción de la teoría de los subconjuntos borrosos a la gestión de las empresas. Santiago de Compostela: Milladoiro.

Kaufmann, A., y Gil-Aluja, J. (1989). Modelos para la investigación de efectos olvidados. Barcelona, España: Milladoiro.

Kosko, B. (1995). Pensamiento borroso: la nueva ciencia de la lógica borrosa. Barcelona, España: Editorial Crítica.

López, M., y Fuentes, L. (2008). Cartera de microcréditos del Sistema Bancario en Venezuela (2002-2005). Visión Gerencial, (2), 355-372. Recuperado de http://www.redalyc.org/articulo.oa?id=465545879012

Luna, K., Sarmiento, W., y Cisneros, D. (2017). Equilibrio de mercado bajo incertidumbre para la fabricación de una bota de dama. Caso Cantón Gualaceo 
Provincia del Azuay. Compendium, 20(39). Recuperado de http://www.redalyc.org/articulo.oa?id=88053976008

Reig, J., y González, J. (2002). Modelo borroso de control de gestión de materiales. Revista Española de Financiación y Contabilidad, 31(112), 431-459. Recuperado de https://www.jstor.org/stable/42781484

Rico, M., y Tinto, J. (2010). Herramientas con base en subconjuntos borrosos. Propuesta procedimental para aplicar expertizaje y recuperar efectos olvidados en la información contable. Actualidad Contable Faces, 13(21). 127-146. http://www.redalyc.org/articulo.oa?id=25718409009

Salazar, R. (2012). El peso mexicano: la gestión de cobertura del riesgo cambiario mediante la Teoría de los Efectos Olvidados. Journal of Economics, Finance and Administrative Science, 17(32), 53-73. Recuperado de http://www.scielo.org.pe/pdf/jefas/v17n32/a06v17n32.pdf

Támara - Ayús, A., Aristizábal, R., y Velásquez, E. (2012). Matrices de transición en el análisis del riesgo crediticio como elemento fundamental en el cálculo de la pérdida esperada en una institución financiera colombiana. Revista Ingenierías Universidad de Medellín, 11(20), 105-114. Recuperado de http://www.redalyc.org/articulo.oa?id=75025069009

Tinto, J., Luna, K., y Cisneros, D. (2017). Teoría de los efectos olvidados en el rescate de la imagen comercial de los artesanos del calzado en el cantón Gualaceo provincia del Azuay, Ecuador. Visión Gerencial, 1, 24-42. Recuperado de http://www.redalyc.org/articulo.oa?id=465549683003 


\section{PARA CITAR EL ARTÍCULO INDEXADO.}

González Astudillo, X., Luna Altamirano, K., Erazo Álvarez, J., \& Sarmiento Espinoza, W. (2019). Recuperación de cartera bajo el enfoque de subconjuntos borrosos. Ciencia Digital, 3(2.3), 156-171. https://doi.org/10.33262/cienciadigital.v3i2.3.595

\section{Ciencia \\ LDigital}

El artículo que se publica es de exclusiva responsabilidad de los autores y no necesariamente reflejan el pensamiento de la Revista Ciencia Digital.

El artículo queda en propiedad de la revista y, por tanto, su publicación parcial y/o total en otro medio tiene que ser autorizado por el director de la Revista Ciencia Digital.
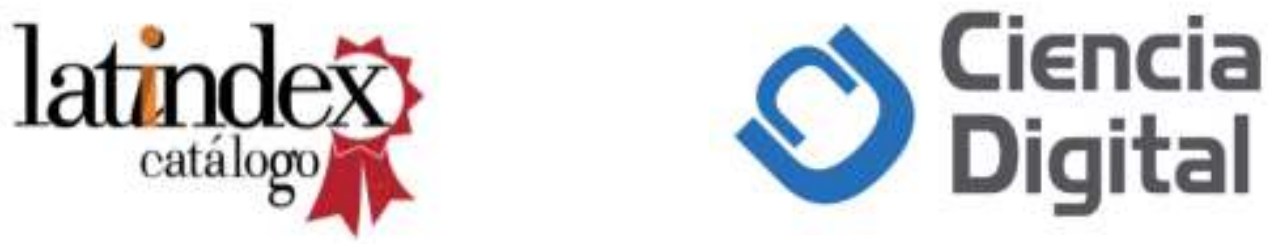